\title{
Conclusiones generales y recomendaciones de las Jornadas de Estudio Euroamericanas de las Entidades Fiscalizadoras Superiores celebradas en Madrid el 17 y 18 de febrero de 2000
}

\section{Preámbulo}

Con ocasión del Congreso de INTOSAI, Montevideo, 1998, se concretó el interés, ya manifestado con anterioridad, de intensificar la comunicación entre las Organizaciones de las Entidades Fiscalizadoras Superiores de Europa (EUROSAI) y de América Latina y el Caribe (OLACEFS), mediante la celebración de un encuentro entre ambos grupos regionales. Esta iniciativa hispanoamericana recibió satisfactoria acogida en la reunión de Praga del Comité Directivo de EUROSAI, en febrero de 1999, en la que el Secretario General de EUROSAI presentó la propuesta inicial del Presidente de OLACEFS. En junio de 1999, en París, el Comité Directivo de EUROSAI aprobó la propuesta de realizar unas Jornadas de reflexión conjunta, en Madrid, coincidiendo con la vigesimosegunda reunión del Comité Directivo de la Organización, en febrero del año 2000.

Estas Primeras Jornadas Euroamericanas, de estudio sobre la cooperación de las Entidades Fiscalizadoras Superiores (EFS) en los procesos de integración y globalización, se han celebrado bajo los auspicios del Tribunal de Cuentas de España, han reunido en Madrid a representantes de las EFS de EUROSAI y OLACEFS y han ofrecido la oportunidad de compartir experiencias y debatir acerca de las perspectivas de colaboración y cooperación en dichos procesos.

Son antiguos los vínculos entre las EFS de EUROSAI y de OLACEFS, que se suman a los que han surgido a través de la participación común en INTOSAI y en sus Comités y Grupos de Trabajo.

Las EFS siempre han reconocido, como recoge la Declaración de Lima, que la cooperación y el intercambio internacional de ideas y experiencias favorecen el cumplimiento de sus funciones dentro de los postulados, emanados de INTOSAI, de independencia, profesionalidad, fomento de la buena gestión financiera, e información a los poderes públicos y a los ciudadanos, mediante la publicación de informes objetivos.

La cooperación presenta, en estos momentos, un nuevo alcance y precisa de renovados impulsos, a la luz de la experiencia de las EFS y de las nuevas realidades políticas, sociales y económicas en que se hallan inmersas, que demandan del control ampliación de esfuerzos y actualización de actividades.

Estas Primeras Jornadas Euroamericanas de las Entidades Fiscalizadoras Superiores han pretendido un acercamiento con- 
creto de nuestras regiones y han permitido a las EFS participantes debatir, compartir experiencias, reconocer nuevos problemas y retos, impulsar el estudio y descubrir nuevas estrategias de cooperación, como se recoge en las siguientes Conclusiones generales y Recomendaciones, que son el resultado de la labor de síntesis de las Ponencias presentadas y de los debates de las diversas sesiones.

\section{Conclusiones generales}

1. La convergencia de valores, principios y objetivos que tiene lugar en los procesos de integración, y la creciente interdependencia, plantean a las EFS nuevas oportunidades de colaboración, en su labor de brindar el adecuado control de la gestión pública que demanda la sociedad civil en los Estados modernos.

La Unión Europea, su ampliación hacia el Este de Europa y el Mediterráneo oriental, la evolución de los sistemas cerrados a sistemas abiertos, los diversos procesos de acercamiento regional en América Latina —como los de MERCOSUR, la Comunidad Andina y el Sistema de Integración Centroamericana-, han generado nuevos ámbitos de actuación para las EFS de Europa y de América y han otorgado una nueva dimensión a la cooperación.

2. ${ }^{\text {a }}$ La cooperación de las EFS de EUROSAI y OLACEFS, en el seno de INTOSAI, permite aprovechar mejor las oportunidades que se presentan ante el nuevo milenio, impulsa la construcción de espacios de integración y constituye una estrategia para fortalecer la ética y consolidar la transparencia de la gestión pública que demanda la globalización.

3. Compartir los valores de los cambios políticos, económicos y sociales que traen consigo los procesos de integración, fundados en la igualdad de derechos y en la diversidad, sitúa a las EFS de EUROSAI y de OLACEFS ante una perspectiva en la que resulta esencial desarrollar estrategias de cooperación encaminadas a afianzar la labor de la fiscalización y a aumentar las sinergias derivadas de la vinculación interregional.

4. ${ }^{2} \quad$ El proceso complejo de la globalización afecta no sólo a la internacionalización de los mercados, a la información, al progreso tecnológico, a la cultura y a las exigencias en materia de derechos humanos, sociales y ecológicos, sino también al control, y reclama de la actuación de las EFS, como elemento de equilibrio en el sistema de poderes de los Estados, el logro de los necesarios consensos para la cooperación y la búsqueda de eficacia.
El nuevo alcance de la cooperación aparece gradualmente, acorde con el nivel de integración en el que actúan las EFS, y permite el funcionamiento de Redes de cooperación simul. táneas entre ellas, con diferentes grados de intensidad.

5. En los nuevos ámbitos institucionales surgidos cuando los procesos de integración, como el de la Unión Europea, adquieren una mayor evolución, se hace necesario gestionar la propia cooperación de las EFS para que su actuación en el sistema integrado pueda resultar eficaz, y esto, en todos los niveles, local o regional, nacional, supranacional y global.

En el proceso de la globalización, los controles públicos, en su nueva dimensión, no pueden considerarse ajenos al ámbito institucional en que se hallan inmersos, ya que resulta determinante de organizaciones y gestiones eficaces en sistemas abiertos, democráticos y de integración en espacios más amplios.

6. ${ }^{\text {a }}$ Es papel de las EFS impulsar la buena gestión financiera, de forma que contribuya, además de al progreso económico, al progreso social y ético, y genere nuevos procedimientos que anticipen los modelos de organización y de gestión pública más adecuados, lo que obliga al control a superar su papel formal para evaluar los riesgos que contienen las propias normas y a constituirse en un elemento impulsor de organizaciones eficientes y de gestiones responsables.

7. ${ }^{\text {a }}$ Las EFS amplían sus ámbitos de actuación, cuantitativa y cualitativamente, tanto en la integración supranacional como en la descentralización de los correspondientes sectores públicos nacionales, y pasa a un primer plano la detección de los fallos y de las responsabilidades de los gestores públicos en materia de organización, métodos de gestión y control interno, a los diversos niveles de la Red de controles.

La integración de controles en una Red constituye la garantía de que el sistema global mantiene la eficacia, permite elaborar informes de fiscalización más actuales, delimita los riesgos derivados de las organizaciones, de las gestiones y de las normas, y otorga mayor relevancia a las EFS en su función de apoyo a los respectivos Parlamentos, con el traslado de resultados que contribuyan a homogeneizar la normativa en materia de controles.

8. ${ }^{a} \quad$ Las EFS necesitan establecer vías de cooperación para llevar a cabo un control eficaz de la aplicación de las ayudas que las Organizaciones Internacionales canalizan en el campo internacional, y superar la mera fiscalización de la contribución económica de cada País miembro.

OLACEFS y EUROSAI pueden constituirse en Organizaciones catalizadoras que faciliten la consecución de la integración de las EFS de Europa y América en el control de la aplicación de los fondos de cooperación internacional y en la consecución de los planes de desarrollo de los respectivos países, 
así como su progreso económico y social, mediante el impulso de cláusulas de reserva de control en los contratos interestatales $y$ de comercio entre ambos Continentes.

9." En América Latina, donde se han iniciado procesos de unión, en distintos grados, sin que hayan culminado en la creación de Instituciones de Control, OLACEFS constituye un ámbito adecuado para que las EFS de los países participantes prevean acciones de control para asumir los nuevos desafíos de la integración y la globalización e impulsar la cooperación que permita configurar y preparar la Red de controles más adecuada a cada etapa.

El acercamiento entre EUROSAI y OLACEFS facilita un nuevo espacio de cooperación común para el enriquecimiento mutuo y la colaboración en materias como el control del medio ambiente, de los proyectos bilaterales y multinacionales, de los fondos de cooperación internacional, de la deuda pública, del fraude y la corrupción, de los procesos de privatización y del funcionamiento de los sectores públicos descentralizados.

10." Las EFS tienen una importante responsabilidad en la integración, cualquiera que sea su nivel formal y las modalidades de colaboración que se prevean. Es papel de OLACEFS y de EUROSAI apoyar esos empeños y cumplir con la función específica de convertir a sus EFS en promotores de la divulgación de las exigencias de control sobre la operatividad de los acuerdos de integración regional, promover el estudio de medios para ejercer sus funciones en el ámbito supranacional, y adecuar los recursos humanos, materiales y tecnológicos a la especialización del trabajo de control.

11. En los procesos de integración que cuentan ya con una Institución de control, como el Tribunal de Cuentas Europeo, es necesario integrar la Red de controles formada por dicha EFS, las de los países miembros de la Unión y —allí donde se simultanean procesos de descentralización de los sectores públicos nacionales- las de las correspondientes Entidades Regionales de Control.

Las zonas concurrentes de fiscalización para el Tribunal de Cuentas Europeo, las EFS nacionales y, en su caso, las Entidades Regionales de Control, reclaman la necesaria cooperación, en la integración y en la descentralización, para que funcione eficazmente el sistema global, trasladando dichas Instituciones a los correspondientes Parlamentos (europeo, nacionales y regionales) los resultados del control sin disfunciones, ni duplicidades.

12." La cooperación en la integración y en la globalización se refiere a todo tipo de actividades destinadas a fomentar la independencia y el status profesional de las EFS y a mejorar la realización de sus funciones, sin limitarse al intercambio de información y experiencias, constituyendo una metodología apropiada para analizar problemas y soluciones comunes y pre- ver la realización de iniciativas conjuntas en el ámbito de la fiscalización.

En los procesos de integración es preciso identificar los nuevos campos de fiscalización para que las EFS, a los diversos niveles de la Red que conforman y actuando en función del conjunto, definan ámbitos, objetivos y metodologías para superar esa «zona gris» de la cooperación estática y pasar a un proceso dinámico de institucionalización de la cooperación, tras el estudio, elaboración de criterios, normas y directivas en los que se recojan valores compartidos.

13. ${ }^{\text {a }}$ Es tarea de las Organizaciones regionales crear las condiciones favorables para una cooperación beneficiosa con las EFS que han evolucionado de sistemas cerrados a sistemas abiertos, promoviendo las oportunidades de interacción técnica $\mathrm{y}$ acercamiento institucional.

14. ${ }^{\text {a }}$ La coordinación, en los países en los que el control se encuentra descentralizado y cuentan con Instituciones de Control de ámbito más reducido que el nacional, tiene que orientarse a evitar disfuncionalidades o duplicidades en la fiscalización concurrente, a potenciar el control, a trasladar los resultados a los correspondientes Parlamentos para mejorar la gestión pública descentralizada y a que los resultados permitan a las EFS nacionales programar eficazmente y realizar fiscalizaciones coordinadas sobre áreas determinadas de la gestión del conjunto del sector público, con resultados de interés para el Parlamento nacional y que permitan depurar las posibles responsabilidades.

La existencia de Instituciones de Control Regionales facilita la evaluación de la eficacia de la gestión pública en todas sus manifestaciones y, mediante una coordinación adecuada, permite evaluar la eficacia del sistema globalmente considerado.

15. La experiencia europea confirma que impulsar la cooperación entre las EFS significa respetar la independencia y los diferentes sistemas legales y de fiscalización. Las EFS deben conceder gran importancia a la cooperación y facilitar los recursos suficientes para llevarla a cabo, mediante la creación de unidades específicas encargadas de la relación con las demás EFS y el establecimiento de procedimientos ágiles de comunicación e información.

Las sólidas relaciones institucionales sirven de apoyo a las Redes de cooperación de las EFS y facilitan el análisis de los problemas comunes y la formulación de propuestas concretas. Su desarrollo exige explorar nuevas iniciativas, compartir recursos de formación profesional, aprobar programas de intercambio de funcionarios, concluir acuerdos cuando se fiscalicen proyectos y organizaciones internacionales e introducir evaluaciones efectuadas por auditores de otras EFS, como elemento de garantía de calidad. 


\section{Recomendaciones}

1. ${ }^{\mathrm{a}}$ El acercamiento de EUROSAI y OLACEFS debería potenciarse mediante seminarios, conferencias y participaciones en actos programados por las dos Organizaciones, en los que debe buscarse la creación de condiciones y el clima favorable para una cooperación mutua beneficiosa y el intercambio de experiencias de fiscalización.

Facilitar información acerca de las metodologías del control que vayan surgiendo en los procesos de integración de la Unión Europea a todas las EFS miembros de EUROSAI y OLACEFS sería muy conveniente para este proceso de acercamiento.

2. ${ }^{\text {a }}$ OLACEFS y EUROSAI deberían desempeñar un papel coordinador entre las EFS de Europa y América, que acerque las realidades y los problemas de fiscalización y de interés común para ambos Continentes.

3. ${ }^{\text {a }}$ EUROSAI y OLACEFS deberían promover la investigación en materia de control encargando la elaboración de conclusiones comunes respaldadas por la experiencia y dando a conocer a las EFS de ambas Organizaciones sus resultados, previa la definición de áreas de interés común para varias EFS de Europa o de América.

4. ${ }^{a}$ OLACEFS y EUROSAI deberían llevar a cabo estudios de identificación de las áreas preferentes de actuación para que el control que las EFS realicen tenga un efecto de prevención sobre cualquier distorsión de los procesos de integración y globalización y pueda anticipar y evitar posibles fallos. Ambas Organizaciones deberían impulsar las acciones regionales conjuntas en materias de trascendencia internacional, como la glo- balización y sus efectos en el control externo público, las alianzas estratégicas de control o la lucha contra la corrupción.

7. ${ }^{\text {a }}$ EUROSAI y OLACEFS deberían potenciar la cooperación mediante el diseño y ejecución de programas de formación, transferencia de tecnología e intercambio de expertos en áreas determinadas, que permitan conocer la experiencia de las EFS más avanzadas, identificar posibilidades en la globalización, crear grupos de trabajo en materias concretas y designar agentes de enlace para la cooperación.

8. ${ }^{a}$ OLACEFS y EUROSAI deberian organizar y llevar a cabo conjuntamente foros de debate, análisis y reflexión sobre materias de control de interés y trascendencia nacional, regional e internacional y promover la comunicación permanente de las actividades de las EFS de ambas Organizaciones en materias de interés común, a través de revistas y boletines profesionales, Internet y correo electrónico.

9. ${ }^{a}$ EUROSAI y OLACEFS deberían impulsar la investigación innovadora, adecuando, creando y perfeccionando conceptos y metodologías, con la finalidad de que la fiscalización resulte eficaz ante las nuevas realidades organizativas del sector público.

Teniendo en cuenta la finalidad de EUROSAI y OLACEFS y los objetivos contemplados en sus Estatutos, ambas Organizaciones deberían impulsar la creación de Cátedras de control en las Universidades, para potenciar la investigación y el conocimiento de las correspondientes metodologías.

10. ${ }^{2}$ OLACEFS y EUROSAI deberían impulsar la cooperación entre EFS, en el marco de los principios de INTOSAI, tanto en el propio ámbito regional en los procesos de integración, como en un espacio intercontinental y global. 